\title{
Mutation rate variation in eukaryotes: evolutionary implications of site-specific mechanisms
}

\section{G. King and Y. Kashi}

Baer et al.'s Review "Mutation rate variation in multicellular eukaryotes: causes and consequences"1 accurately conveys the tenor of most literature on mutation rate evolution. Even while recognizing that mutation rates can vary dramatically from site to site within a genome, this literature mostly ignores or minimizes the special characteristics and consequences of site-specific mutational mechanisms. Therefore, to the authors' list of "five critical questions" for future investigation, we would like to propose a sixth: to what extent can elevated site-specific mutation rates vary (and evolve) independently from low average nucleotide-substitution rates?

Following historical precedent, Baer et al. ${ }^{1}$ consistently refer to "the mutation rate" as if a single value could adequately summarize the complex outcome of many diverse mutational mechanisms. However, such a simplistic statistic should no longer be tolerated, at least not without precise qualification such as "average rate of nucleotide substitutions within protein-coding sequences”. Even then, somatic mutability of antibody genes demonstrates that localized hypermutation can be exploited by adaptive evolution ${ }^{2}$. Site-specific sources of germline mutation are also familiar, as evidenced by the prolific variation that is produced by simple sequence repeats (SSRs; microsatellites and minisatellites) ${ }^{3}$.

Although Baer et al. ${ }^{1}$ acknowledge the remarkably high mutation rates of SSRs, they do not discuss the profound implications of such site-specific mutability for understanding mutation rate evolution. Phenotypic effects of SSR mutations include reversible on-off switching and quantitative variation in many aspects of gene function ${ }^{3-8}$. Such effects need not be predominantly deleterious, especially when adaptation is suboptimal, as in variable environments ${ }^{9}$. But an assumption that "the vast majority of mutations with observable effects are deleterious" has informed most discussion of mutation rate evolution throughout the past century ${ }^{1}$. Traditionally, theoretical discussion also assumes that recombination in diploid organisms must eventually separate 'mutator alleles' from resulting mutations, thereby preventing mutators from 'hitchhiking' on selection for beneficial mutants ${ }^{1}$. However, recombination cannot unlink site-specific mutational mechanisms, such as those based on SSRs, from the mutations that they generate. Hence, selection for any beneficial mutation at a mutable site must also, indirectly, favour the site's intrinsic mutability.

The best evidence for positive selection of sites with high mutation rates comes from the SSR-based contingency genes of haploid microorganisms ${ }^{10}$. In eukaryotes, the reported abundances, genomic distributions, phylogenetic conservation and patterns of variation for SSRs are also strongly suggestive of positive selection ${ }^{4,6,7}$. The utility of SSR mutability for adaptive evolution has already been implicated in several cases, including skeletal evolution of domestic dogs ${ }^{5}$, temperature compensation of the Drosophila melanogaster circadian clock ${ }^{11-13}$ and social behaviour of voles ${ }^{14}$, among others ${ }^{7}$. Whether such mutable sites somehow prevail in spite of their high mutability (as conventional theory requires), or whether indirect selection for their high mutability is the reason for their prevalence ${ }^{8,9}$, remains to be established. In either case, the special characteristics of site-specific mutational mechanisms deserve attention in any future consideration of mutation rate evolution.

D. G. King is at the Department of Anatomy and Department of Zoology, Southern Illinois University Carbondale, Carbondale, Illinois, USA.

Y. Kashi is at the Department of Biotechnology and Food Engineering, Israel Institute of Technology (The Technion), Haifa, Israel.

Correspondence to D.G.K e-mail:dgking@siu.edu

Baer, C. F., Miyamoto, M. M. \& Denver, D. R. Mutation rate variation in multicellular eukaryotes: causes and consequences. Nature Rev. Genet. 8 , 619-631 (2007).

2. Beale, R. \& Iber, D. in The Implicit Genome (ed. Caporale, L. H.) 177-190 (Oxford Univ. Press, Oxford, 2006).

3. Kashi, Y., King, D. G. \& Soller, M. Simple sequence repeats as a source of quantitative genetic variation. Trends Genet. 13, 74-78 (1997).

4. King, D. G. \& Soller, M. in Evolutionary Theory and Processes: Modern Perspectives (ed. Wasser, S. P.) 65-82 (Kluwer Acad. Publ., Dordrecht, 1999).

5. Fondon, J. W. 3rd \& Garner, H. R. Molecular origins of rapid and continuous morphological evolution. Proc. Natl Acad. Sci. USA 101, 18058-18063 (2004).

6. King, D. G., Trifonov, E. N. \& Kashi, Y. in The Implicit Genome (ed. Caporale, L. H.) 77-90 (Oxford Univ. Press, Oxford, 2006).

7. Kashi, Y. \& King, D. G. Simple sequence repeats as advantageous mutators in evolution. Trends Genet. 22, 253-259 (2006).

8. King, D. G. \& Kashi, Y. Indirect selection for mutability. Heredity 99, 123-124 (2007)

9. Levins, R. Evolution in Changing Environments. (Princeton Univ. Press, Princeton, 1968).

10. Bayliss, C. D. \& Moxon, E. R. in The Implicit Genome (ed. Caporale, L. H.) 54-76 (Oxford Univ. Press, Oxford, 2006).

11. Sawyer, L. A. et al. Natural variation in a Drosophila clock gene and temperature compensation. Science 278, 2117-2120 (1997).

12. Zamorzaeva, I., Rashkovetsky, E., Nevo, E. \& Korol, A Sequence polymorphism of candidate behavioural genes in Drosophila melanogaster flies from 'Evolution Canyon'. Mol. Ecol. 14, 3235-3245 (2005).

13. Sawyer, L. A. et al. The period gene Thr-Gly polymorphism in Australian and African Drosophila melanogaster populations: implications for selection. Genetics 174, 465-480 (2006).

14. Hammock, E. A. D. \& Young, L. J. Microsatellite instability generates diversity in brain and sociobehavioral traits. Science 308, 1630-1634 (2005). 